\title{
Survey of crystal singular optics
}

\author{
Angelsky, O.V.; Zenkova, C. Yu.; Hanson, Steen Grüner
}

Published in:

Introduction to Singular Correlation Optics

Publication date:

2019

Document Version

Peer reviewed version

Link back to DTU Orbit

Citation (APA):

Angelsky, O. V., Zenkova, C. Y., \& Hanson, S. G. (2019). Survey of crystal singular optics. In O. V. Angelsky (Ed.), Introduction to Singular Correlation Optics (Vol. PM295). SPIE - International Society for Optical Engineering.

General rights

Copyright and moral rights for the publications made accessible in the public portal are retained by the authors and/or other copyright owners and it is a condition of accessing publications that users recognise and abide by the legal requirements associated with these rights.

- Users may download and print one copy of any publication from the public portal for the purpose of private study or research.

- You may not further distribute the material or use it for any profit-making activity or commercial gain

- You may freely distribute the URL identifying the publication in the public portal

If you believe that this document breaches copyright please contact us providing details, and we will remove access to the work immediately and investigate your claim. 


\section{INTRODUCTION TO CORRELATION SINGULAR OPTICS}

\section{Chapter 6. Survey of crystal singular optics}

by O.V. Angelsky, C.Yu.Zenkova and S.G.Hanson

Keywords: polarization, anisotropic media, birefringence, polarization singularities, orbital and spin angular momenta, white-light vortices, uniaxial and biaxial crystals, liquid crystals 


\section{Chapter 6. Survey of crystal singular optics}

\subsection{Initial approaches of the crystal singular optics}

Among the directions of modern optics finding out an application in design of optical nano-tweezers and nano-manipulators, one can emphasize the use of the singular optics approaches to anisotropic media. It is known that singular optics deals with the light beams supporting wave-front edge dislocations and optical vortices (screw dislocations) as well as polarization singularities. For that, optical vortices form specific intensity distributions capable to trap, transport and control of orientation of microparticles. New feasibilities of singular optics have been revealed just due to using optically anisotropic crystals. The obtained knowledge enriches our knowledge of the properties of laser beams and yields new promising applications of them, in part for implementation of optical tweezers and optical telecommunications. One more direction of singular optics is the nonlinear singular optics using nonlinear crystals with singularities providing creation of optical solitons, i.e. solitons in optical vortices.

Generally, applicability of the term "crystal" occurs be much wider than for designation of conventional anisotropic media [1]. So, the notion 'crystal' is applicable for any anisotropic medium including chiral one: liquid crystals or plastics such as overheadprojector transparency foil [2] under nonmagnetic anisotropy [3].

The classical linear wave optics of homogeneous nonmagnetic crystals was studied throughout many decades, since the initial approaches [4-7] (see also review [8]) based on the Maxwell's equations up to the classical optics of crystals and modern theory of nucleation and propagation of singularities in anisotropic media with optical activity (chirality) and dichroism (absorption)) [1]. Propagation of plane waves through a birefringent uniaxial media was considered in details in the beginning of the 20th century in the framework of the Fresnel's formulae [4]. Classical crystal optics provides beautiful illustrations and extensions of the generic polarization singularities of optical fields, in which there is much current interest [9-12]. This is the part of a wider exploration of optical singularities, for example, phase dislocations [13-15] and caustics and associated diffraction [16, 17].

Unique property of birefringent crystals consists in their ability to transform the Gaussian beam to a singular one yielding the feasibilities for control the parameters of an optical vortex propagating along optical axis. Gaussian beam propagating perpendicularly to the optical axis of crystal is divided into two beams, ordinary and extraordinary. The ordinary beam propagates through anisotropic medium as in isotropic one. Anisotropic diffraction in crystals leads to changing transversal cross section of the extraordinary Gaussian beam resulting in elliptical deformation of this beam (the vortex-beam 'precession' in a rotating uniaxial crystal [18]).

One of the early approaches provided description of propagation of an optical wave through anisotropic crystalline medium was based on the concept of the angularspectrum representation [19]. Within the first approximation, a crystal is considered to be uniaxial, infinite and homogeneous, and the medium is believed to be conducting and possessing temporal dispersion. Under this approximation, the permeability and conductivity are taken to be scalar constants. Spatial-temporal Fourier transform of conditional sources localized in a crystal or finding out a plane wave amplitudes is used taking account of orientation of the crystallographic axes of a crystal. In contrast to the traditional approaches using the Green's functions, here the behavior of non-plane 
waves is determined in terms of plane ones. Representation of non-plane waves in anisotropic medium has been introduced by Carrier [20], Bunkin [21], Lighthill [22]. In investigations of Lighthill, the radiated electromagnetic field is considered by applying the combination of the stationary phase technique and generalized functions. In parallel, it has been proposed to divide a beam in the media in two components associated with TE and TM fields along the optical axis with a point dipole oriented along or perpendicularly to the optical axis as the conditional source, what restricts applicability of this method. The base of the mentioned approaches is the fundamental notions on the Gaussian beams or on dark hollow beams with circular and rectangular symmetry [20].

Demonstration of propagation of a light beam in anisotropic medium is also possible using the paraxial approximation based on the notion of homogeneous anisotropic medium with small spatial variations of refraction index [23].

In a uniaxial crystal, a beam propagating along an arbitrary direction is always a superposition of an ordinary and extraordinary parts, which are rigorously uncoupled, and both contribute to each transverse component of the beam. The difference between the diffraction of the extraordinary and the ordinary parts gives rise to the coupling between the transverse components of the field. These features are physically interpreted as resulting from the combined effects of the plane-wave structure in uniaxial crystals and the paraxiality, according to which the dominant role in beam propagation is played by the plane waves [24].

The interest in the ability of crystals to generate optical vortices is caused by the feasibility to form the beams with specified content of vortices whose spatial positions are simply controlled. Singular beams in a uniaxial crystal acquire the properties non intrinsic for them in other optical systems. So, a uniaxial crystal performs multiplexing and dividing that results in changing the vortex topological charge. The magnitude of the charge of transformed vortex depends on both the structure of the initial beam and its polarization [25, 26]. A uniaxial crystal can generate polychromatic ('white-light') vortices. At the same time, the disturbed optical vortices in a crystal transferred by a singular beam can also be undergone to the mathematical operation of summation as the result of changing their fine structure. The each disturbed vortex is the sum of two oppositely charged partial vortices, whose weights can be smoothly controlled by applying the external polarization disturbance. The system provides a control of the positions of the coupled optical vortices as well as their angular momenta, what is used in devices for trapping, transporting and mutual orienting microparticles.

Physical mechanisms of formation of complex vector fields in crystals provide transformations of homogeneously polarized beams propagating along the optical axis of a crystal or at small angle to it [27]. When a laser beam propagates at small angle to the perpendicular to the optical axis of a crystal, the parameters and characteristics of a beam are changed considerably [18]. These peculiarities of vortex beams in anisotropic media are of importance in implementing optical tweezers and singular optics microscopy. Twirling of the lines of the resulting optical current stipulates for the transfer of the beam's orbital angular momentum (OAM). In the simplest scalar case, the OAM is proportional to the vortex topological charge. The presence of the OAM of an optical beam plays the key role in devices of transfer and processing of superdense data files. Rotation of a crystal in respect of the optical axis provides diverse configurations of polarization singularities at the crystal output [28]. 
The mentioned above shows the feasibilities for forming and controlling the optical vortices that leads to reaching the potentialities of the devices for control of micro and nano-objects.

\subsection{Peculiarities of propagation of optical beams through anisotropic crystals}

One of the methods enabling to describe propagation of a beam perpendicularly to the optical axis of anisotropic crystal is based on the attracting the notion of the Fresnel's surfaces. Within this approach, the beam is not divided into two beams with different form and propagation direction, but is only undergone the phase and group velocities of propagation. For that, the last investigations demonstrate [18] that a common paraxial Gaussian beam is nevertheless divided into two beams, so that the ordinary beam keeps its cross-section form, while the extraordinary beam is elliptically deformed. The magnitude of deformation of the transversal cross-section is determined by the crystal birefringence.

Astigmatic transformation of a singular beam, in part its elliptical deformation in a free space or in homogeneous medium, changes considerably the structure of optical vortices due to high sensitivity of the phase of beam to any, even weak changes of the beam form. It is naturally that the changing parameters of anisotropic medium have essential influence upon propagation of a vortex beam [18].

When a homogeneously polarized laser beam propagates through media with inhomogeneous refraction index, the set of specific points arise, forming typical distribution irrespectively of origin of inhomogeneities. Such specific points of vector field are the disclinations $[7,17]$ playing the key role in formation of optical vortices into the beam propagating through a uniaxial crystal. To mark out such points, one must consider those of them for which the structural stability of vector light field takes place. Propagation of such beams through a crystal results in inhomogeneous at cross-section polarization. Special points or lines at this vector field where the circular polarization is clockwise or counter-clockwise have been are referred to (following M. Berry [27]) as "star", "lemon", "monstar" (from "lemonstar", something intermediate between the first and second states). These singularities, being structurally stable configurations of a field, enable to detect the changes in the field as a result of external disturbance [28]. The regions of singularities kind of the star, the lemon and the monstar at complex vector field of polarization distributions at the beams propagating through a birefringent uniaxial media can be predicted using the approximation of plane waves within the paraxial wave equations $[29,30]$.

The mentioned three configurations differ in the number and orientation of the lines, at the each point of which the major (minor) axes of neighboring ellipses are oriented to $C$ point, as it is shown in Fig. 6.1. So, the star (S) contains three pairs of the forming lines, lemon (L) - one, and monstar (M) - three, oriented asymmetrically. At crossing $\mathrm{C}$ point, the forming lines for the major and minor axes of the polarization ellipses are changed each to other. Under circumference $C$ point, the ellipses rotate by $\pm \pi$, what determines their topological index or the number of the revolutions of the polarization ellipse major axis when circling around the polarization singularity: the star has the index equal $s=-1 / 2$, while for lemon and monstar $s=1 / 2$. 
(a)

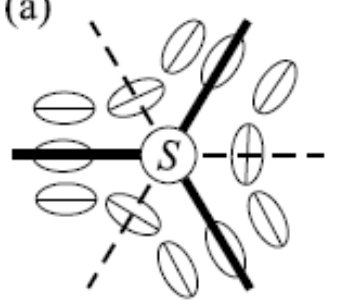

(b)

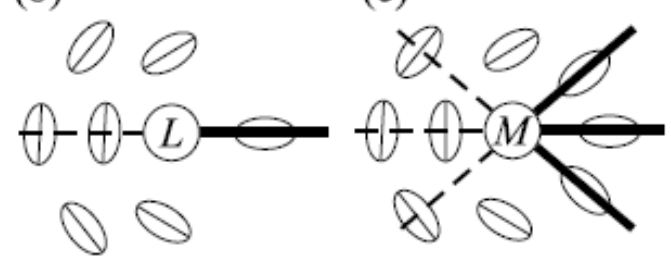

Fig. 6.1. Morphologic forms of polarization neighborhood of $C$ points [31]: a) star (S), b) - lemon (L), C) - monstar (M). Major and minor axes of ellipses are oriented along solid and dashed lines, respectively.

The considered singularities are born and annihilate in pairs, to say S-M, due to the conservation law for the topological index and topological similarity [31]. In presence of any external influence, the lemon can be transformed into the monstar and vice versa. The birth and annihilation of $C$ points follow to one of two scenario depending on orientation of the major or minor axes of the polarization ellipse. For example, before the birth of $C$ point local increasing of ellipticity takes place, what is larger than the average magnitude of ellipticity for remove ellipses. Similar "residual structure" is observed after singularity annihilation, what can be used for data transfer with encoding by $C$ points at laser beam [31]. Even under strong disturbances of a field initiating annihilation of $\mathrm{C}$ points, the reconstruction of the initial field configuration is possible, with some probability.

Later, it has been revealed [6.33] new type of polarization singularities arising under propagation of light through a birefringent crystal due to transformation of optical vortex at the crystal output. A homogeneously polarized incident beam is divided in a crystal into two orthogonally linearly polarized beams propagating in different directions. Superposition of these two waves results in complex polarization distribution that can be described in terms of polarization singularities, viz. as the sets of $L$ surfaces where the polarization is linear and $C$ lines where the polarization is circular [17, 32]. These singularities intersect the transverse plane of observation along $L$ lines and $C$ points, respectively. Conventionally, such polarization singularities arise in speckle-fields or in a near-field micro-optics [33-36], where, in general, polarization at transversal crosssection is inhomogeneously elliptically polarized. $L$ lines (at the specified cross-section of a field) and surfaces (in three dimensions) separate regions of opposite handedness [17], and $C$ lines and points are characterized by a disclination index $\pm 1 / 2$.

It is of interest that propagation through a birefringent crystal of a monochromatic, initially homogeneously polarized beam supporting optical vortex results in forming of inhomogeneously polarized field containing the net of singularities with a general structure kind of a double helix of $C$ lines and $L$ surfaces. These structures are 3D ones, see Fig. 6.2. Beside of conventionally considered transversal linearly polarized components resulting from birefringence in a crystal, the third (longitudinal) polarization component occurs that depends on the phase shift $\Lambda$ between two orthogonal polarization components determined by an optical path difference due to propagation through the crystal, as the result of superposition of orthogonal polarization components. Such configuration of singularities is possible, for example, when linearly polarized $L G_{0}^{1}$ beam propagates through KDP crystal. 

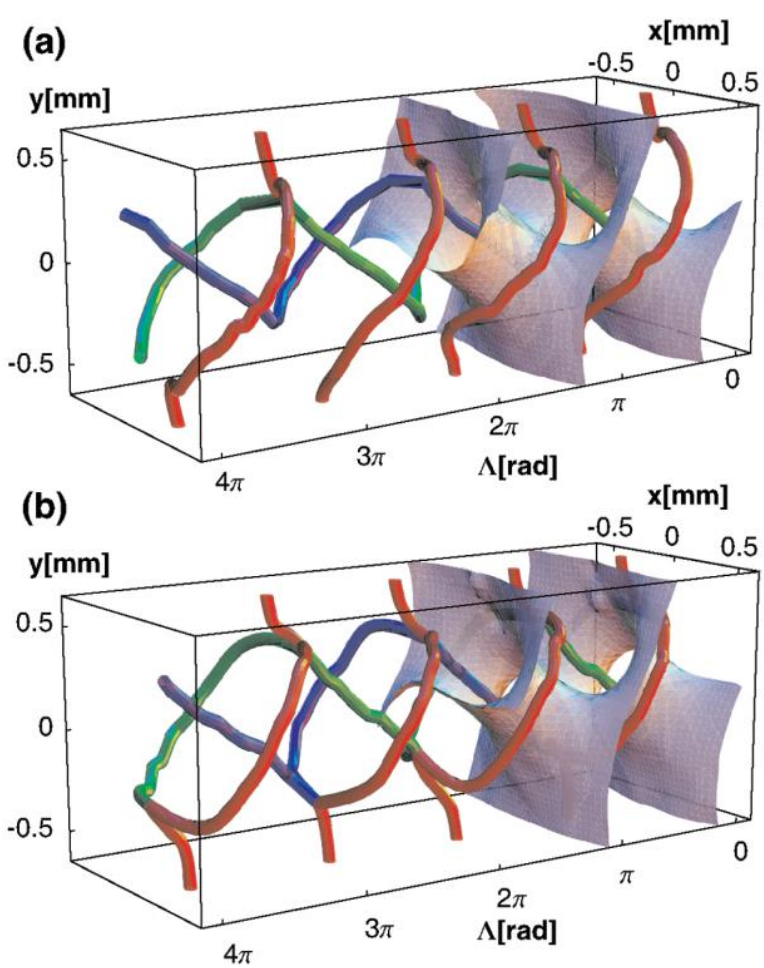

Fig. 6.2 [34]. The net of polarization singularities ( $L$ surface and $C$ lines) in $(x ; y ; \Lambda)$ space for initial linear polarizations: (a) $\alpha_{i n}=40^{\circ}$ and (b) $\alpha_{i n}=45^{\circ} . \Lambda$ changes from 0 to $4 \pi$, the $L$ surface is plotted from 0 to $2 \pi$. In (a) all $C$ lines are disconnected; in (b) reconnections occur.

More complex singular structures in crystals possessing optical activity and dichroism and using polychromatic light can be also considered.

\subsection{Formation of fine structure of optical singularities in anisotropic media with dichroism and absorption}

For extending the approaches and notions of singular optics on the base of the classical theory of propagation of optical fields through crystal, one must to take into account influence, beside of anisotropy, the optical activity (gyrotropy). For that, one must take into account that a crystal is chiral and possesses absorption ability (dichroism). Description of the optical properties of crystals is considerably complicated, when biaxial birefringence is added by chirality and dichroism. Three types of polarization singularities are realized in this case [32].

The type 1 - the singular axes for which the magnitudes of the refraction indices are equal. For transparent non-chiral crystals these axes coincide. In general case, the singular axes separate the regions with different refraction indices. Due to absorption, the each optical axis is splitted, and the magnitude of splitting is determined by the magnitude of chirality, what appears or disappears as chirality occurs and increases. Note, for non-chiral transparent crystals the points into s-space form the optical axes; the linear dichroism splits the each optical axis into the pair of singular axes, which are combined again as chirality increases. Singular and optical axes are characterized by different geometries [1]. 
The type 2-C points, at which polarization is circular (clockwise or counter-clockwise), independent on chirality. The each $\mathrm{C}$ point is characterized by the topological index, whose magnitudes are: +1 (uniaxial transparent non-chiral crystal), $+1 / 2$ (transparent non-chiral or transparent chiral, or dichroic chiral crystal), or $+1 / 4$ (dichroic non-chiral crystal). C points are characterized by circular (clockwise or counter-clockwise) polarization, for that one of the circularly polarized components disappears here. Similarly to the case of anisotropic media without chirality, the each $\mathrm{C}$ point is singular in specific manner, viz. it is characterized by the topological index describing the polarization ellipses in the vicinity of this point. The topological index is determined by the number of rotation of ellipses around their centers in the vicinity of $C$ point. In transparent non-chiral crystal, $\mathrm{C}$ points lie at the optical axis. If a crystal is chiral and C axes are fixed, the singular axes disappear.

The Type $3-L$ lines, along which polarization is linear; these lines divide a space into the regions with right- and left-handedness including $C$ points. In presence of absorption but in absence of chirality, the positions of $L$ lines are kept unchangeable. For chiral crystals the 'plus' and 'minus' $L$ lines are different.

Specific polarization singularities observed in chiral anisotropic media can be elaborated by applying the stereoscopic projection technique based on the properties of isolated plane waves constituting the beam that provides obtaining a fine structure of the polarization singularities in such crystals $[37,38]$.

\subsection{Formation of optical quadrupoles in optical beams inclined to the optical axis}

Among variety of peculiarities of vortex transformations in singular beams propagating through optically homogeneous uniaxial crystal, one can observe the set of unconventional effects. There are topological properties of the polarization singularities at Gaussian beams propagating through such structures, which manifest themselves in formation of specific distribution of singular beams with complex structure, so-called topological multipoles consisting of several spatially separated vortices.

One of the recently proposed new approaches for creation of singly charged optical vortices consists in the use of a uniaxial crystal, through which a linearly polarized Gaussian beam propagates in the x-direction.

To describe the propagation of paraxial beams slightly inclined to the optical axis of a uniaxial crystal, one uses the model of two linearly polarized beams generated in a crystal. Even small deviation of the Gaussian beam from the optical axis of a uniaxial crystal results in generation of the polarization singularity shifted in respect to the axis and split into two 'lemons'. Such shift is caused by some doubly charged optical vortex at one of the components of a circularly polarized beam out off the crystal axis, and splitting is associated with the generation of two singly charged vortices. For that, the transversal cross section of a beam changes as inclination of a beam in respect to changing the beam propagation directon.

Conventional plane-wave approximation is insufficient for description of influence of the beam inclination on the splitting a circularly polarized beam into two linearly polarized ones. Initially, both the ordinary and extraordinary components of the polarized beams propagate together, along the axis of the inclined beam that is accompanied by the reactions of birth and annihilation of optical vortices. Note, the optical vortices are mixed 
in such a manner that one cannot associate any vortex with some specified component. As a beam propagates, vortices are redistributed being attributed to the ordinary and extraordinary beams and detected at both circularly polarized components [38].

Topological charges of the components of off-axis beams resulting from the splitting into components are different from the charges of the same components for the beam propagation along the optical axis. In some cases, nevertheless, arising optical vortices with the same topological charges is possible [39]. The doubly charged vortex disappears in the combined beam taking away the OAM. Moreover, the beam is depolarized losing in this way its spin angular momentum. An inclined beam acquires new properties, such as generation of singly charged vortices and the beam quadrefringence. The beam quadrefringence results from splitting the inclined beam into two ordinary and extraordinary beams forming two doughnut beams. For that, the transversal shift of a beam at one of the circularly polarized components is accompanied by arising of the additional angular momentum for conserving balance of the whole angular momentum flow.

Thus, deformation of a beam inclined to the optical axis of a crystal is accompanied by nucleation of a topological quadrupole, viz. the structure consisting of four vortices with opposite single charges imprinted in four individual beams of the complex, combined beam. This effect is referred to as the vortex quadrefringence (from quadru-refringence) [39]. In this case, the y-component arises. A beam propagating through a crystal becomes to be elliptically polarized, with intensity re-distributed among the polarization components. Four zones of zero intensity are formed, which appear symmetrically on the ring surrounding the beam origin with the phase change by $2 \pi$ under circumference of the point of amplitude zero. Thus, four vortices are formed, the each of which is attributed with the certain beam. These four vortices have the alternative topological charges and form the topological quadrupole. Interference of two inclined singular beams is accompanied by the reactions of birth and annihilation of optical vortices with deformed structure of a beam at one of circularly polarized components. Small (at level of a wave length) transversal shift of a beam enables to estimate this effect as conventional birefringence of the vortex beam. Any change of the direction of propagation of a beam in respect to the fast axis leads to deformation of the beam structure manifesting itself into changing distribution of polarization singularities [39].

The polarization distribution formed in such manner consists of polarization ellipses with different handedness covering the polarization map, while polarization became linear on the $L$ lines separating the regions of elliptical polarization (Fig. 6.3 [40]).
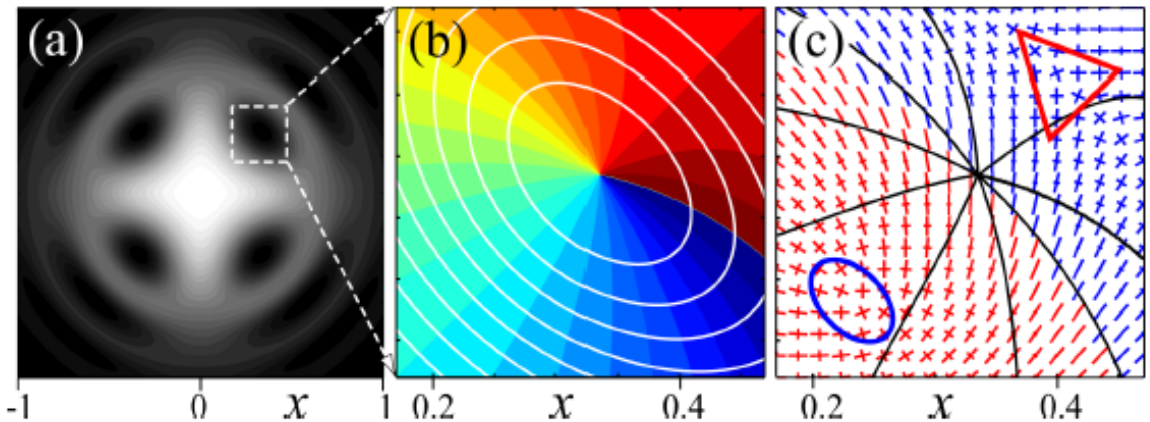

Fig. 6.3 [40]. Numerically calculated (a) intensity and (b) phase profiles of the $x$ polarized field component; (c) The polarization map of the total transmitted field with ellipses of the left-hand (red) and right-hand (blue) elliptical polarizations indicated by their major axes. Blue ellipse and red triangle indicate the 'monstar' and 'star' polarization singularities. 
One can observe two isolated $\mathrm{C}$ points where the field is circularly polarized (depicted by the oval and triangular). These $\mathrm{C}$ points are the 'monstar' and the 'star'-type polarization singularities, respectively. Detecting such singularities at vector field is implemented using a polarization filter suppressing clockwise or counter-clockwise polarization.

The phase distribution of the $x$-polarized component is rather complex, containing the contour lines that form a "spider" pattern with uncertainty at the central point (Fig. 6.3 (c)). Placing a polarizer behind a crystal enables to convert a polarization singularity into optical vortex. Four resulting vortices are assembled in a topological quadrupole [4043].

The effects discussed above can be unambiguously interpreted within the framework of the matrix model [40], which provides description of both fine polarisation structure of the vortex beam in the intermediate range of inclination angles, beginning from the onaxis propagation (excluding the beam propagation along the optic axis) up to the angle of beam splitting (a so-called undistinguished border), and takes into account a degenerate case of the on-axis beam propagation and the asymptotic beam splitting in the crystal.

\subsection{Formation of white-light vortices in crystal singular optics}

The feasibility to generate optical vortices in polychromatic EM radiation ('white-light' vortices) has been firstly demonstrated by reproduction of the wave caustics resulting from scattering of a white light by a rifled glass [44]. The regular 'packing' of isolated vortices provides an analogy to the crystalline lattice. The vicinity of the each vortex is colored, being essentially different from the distribution of colors in a rainbow. However, this technique does not provide the formation of isolated white-light vortices. It has been proposed in papers $[45,46]$ to create white-light vortices under propagation of a beam through a uniaxial crystal and a polarization filter. This approach presumes control of a vortex position at the beam. Vortices formed in such a way are characterized by a fine structure differing from the structure of monochromatic vortices. Degenerated vortex nucleating at the crystal axis in absence of the external polarization disturbance is split into two isolated vortices due to influence of the external disturbance. The contours of vortices are blurred as a distance from the axis increases due to depolarization of a beam. The disturbed vortices acquire new properties. It has been shown experimentally $[45,46]$ that the vicinity of the disturbed vortex is of depleted coloring essentially differing from coloring of the diffraction pattern minima.

Fine structure of such white-light vortices is characterized at three levels, viz. the polarization structure, the topological charge structure, and the color structure. The main parameters describing the white-light vortices are experimentally determined characteristics, such as the orbital parameter or the ellipticity of the vortex core, the probability to meet such a vortex state and the noise of the vortex core or the polarization degree in the given space point. The color gamut in the vicinity of a phase singularity is the additional characteristics of the white-light vortex [47].

Thus, the remarkable property of birefringent crystals consists in their ability to form symmetrical distributions of the polarization states with degenerated $\mathrm{C}$ points at the beam axis with the topological index $s=1$, which are not depending on a wave length, that enable to create of polychromatic (white-light) optical vortices. 


\subsection{Propagation of optical beam in biaxial crystals}

The propagation of light along an optical axis of a crystal depends on the symmetry of the crystal. The optical beam propagating along the optical axis of a uniaxial crystal is not undergone any transformations. But if a beam propagates through optically biaxial crystal, as KTP or LBO, it is transformed radically. This is the phenomenon of conical refraction. The light beam does not change in a uniaxial crystal but radically transforms its intensity and polarization distribution in a biaxial crystal, which may also possess chirality (optical activity) [48]. In such crystals, a beam propagates within a hollow cone transforming at output into hollow cylinder. The beam structure is determined by the diffraction effects under propagation through a crystal being depending on the distance from a crystal, the ratio of the cylinder radius to the transversal cross-section magnitude of a beam, as well as on the chirality of a beam, see Fig. 6.4.

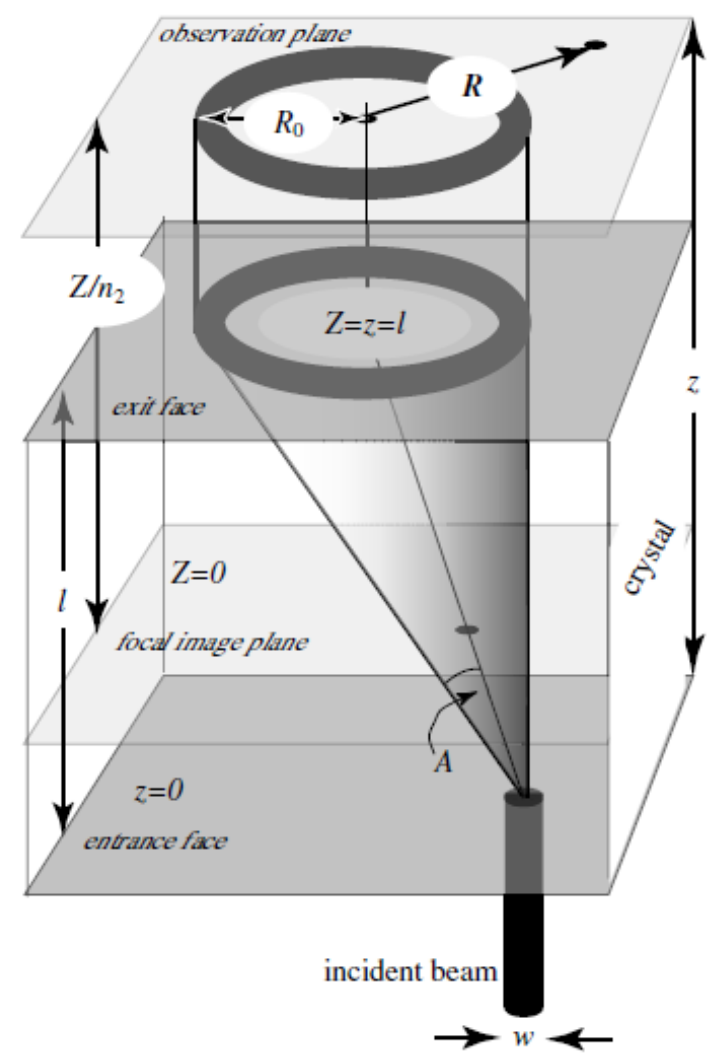

Fig. 6.4 [48]. Formation of the system of rings due to conical diffraction; dark ring has radius $R_{0}$ for an incident beam of cross-section $w$ propagating through biaxial crystal of thickness $l$.

For any biaxial crystal, irrespectively to its optical activity, the total angular momentum of the beam at the output of a plate differs from the angular momentum at the incident beam. Thus the beam must acquire at a crystal the torque in respect to the direction of the incident beam depending on crystal parameters. The torque can arise owing to the non-coincidence of the axes of conical and cylindrical beams with the direction of propagation of the incident beam. It leads to arising the torque in respect to the axis localized at the plane of a sample and perpendicular the incident beam. Its magnitude exceeds considerably the torque associated with the change of the state of polarization. As a result, transformation of the beam at the crystal output differs from one at propagation of the same beam through optically uniaxial crystal [48]. 


\subsection{Anisotropic crystals as the media for generation of optical vortices}

Generation of optical singularities, both phase and polarization ones, is possible in the case when two, three or more number of plane waves interfere [49]. Optical vortices with non-zero azimuthal energy flows are the phase singularities being characterized by the OAM, what predetermines applications of such beams in micro-manipulations, microscopy, astronomy etc.

Complex systems of birefringent crystals spatially ordered at the transversal plane of a beam, such as calcite, provides the possibility of selective manipulation of a microobject depending on the position of a crystal. Combining the polarization singularities enables to increase the possibilities of optical manipulation of various microobjects. So, ellipticity of a beam into birefringent crystal causes the rotating polarization singularities dependent on the crystal orientation. The change of the state of polarization of the incident beam causes changing the direction of rotation. Illumination of a crystal by the two-component beam leads to specified direction of the crystal rotation depending on the state of polarization of a beam [50].

Azimuthal breaking of the initially smooth wave front causes generation of an optical vortex due to refraction, diffraction, anisotropic phase manipulation and birefringence in anisotropic media. For that, generation of optical vortices in the uniaxial crystals has some advantages in comparison with conventional methods, to say using computergenerated holograms, limited by low diffraction efficiency of diffractive optical elements and the narrow spectral bandwidth constraint of spiral plates. The mentioned effects are minimized for the spin-to-orbital angular momentum coupling in anisotropic materials whose spectral dependence scales as the birefringence dispersion.

Using the uniaxial crystals enables, for the on-axis propagation of linearly polarized fundamental Gaussian beams, to generate on-axis isolated single charge vortices with the efficiency $75 \%$. In the uniaxial crystals, generation of white-light optical vortices is accompanied by chromatic dispersion, shift of beams and rainbow colouring due to wavelength-dependent diffraction effects [51].

The use of liquid crystals for generation of optical vortices is of especial interest. For example, the spatial light modulators (SLM) on the base of 2D mosaic liquid-crystal (LC) massifs are the efficient tool for generation of optical vortices. Anisotropic media kind of LCs are used for making the phase plates in the form of symmetrical devices generating the optical vortices due to the setting of the state of polarization of the incident beam and exploiting of the radially symmetrical birefringence that results in generation of vortices in one LC drop. The drops prepared using specified configuration of the nematic LC enable to control the orientations of the director and the local optical axis with the spherically symmetrical 3D distribution of the director in a drop. This simple 3D approach is the competitor to the expensive multilevel 2D lithography technique providing new feasibilities for creation of dense regions of optical vortices in anisotropic colloid.

Let us consider an example of the mentioned system consisting of two LC drops closely positioned to each other. Being illuminated by laser radiation, the drops are the secondary radiators with the same state of polarization forming an interference pattern associated with the azimuthally invariant wave front with the spin-dependent azimuthally changed phase. The same system can be used for generation of polychromatic (whitelight) vortices. 
Thickness of a material, the magnitude of birefringence and the system geometry determine the condition of the optical vortices generation. The uniqueness of this approach is that a beam is captured at the axis and conserves its radial symmetry. As a result, such system ensures generation of the axial optical vortices without using additional high-sensitive optical elements associated with other techniques. For that, high sensitivity of LC molecules to external fields (electric, magnetic, thermal, chemical or optical) provides new feasibilities in solving the wide variety problems of adjusting and control [52].

LCs are also used for design of so-called light valves for generation of optical vortices. LC light valves have been designed on the base of defects of the texture of LC within the valve. Liquid crystal light valve (LCLV) is composed of a photoconductive layer, i.e. made of a slab of bulk monocrystalline $\mathrm{Bi}_{12} \mathrm{SiO}_{20}$ (bismuth silicon oxide, $\mathrm{BSO}$ ) with the outside surface coated with indium tin oxide (ITO), and a thin glass plate with ITO deposited on the inside surface [53]. A circularly polarized Gaussian probing beam illuminates a LCLV that leads to increasing conductivity at the spot centre (Fig. 6.5). As a consequence, the distribution of voltage at LC takes a bell-like form with some alternating voltage from the centre of a beam to its periphery.

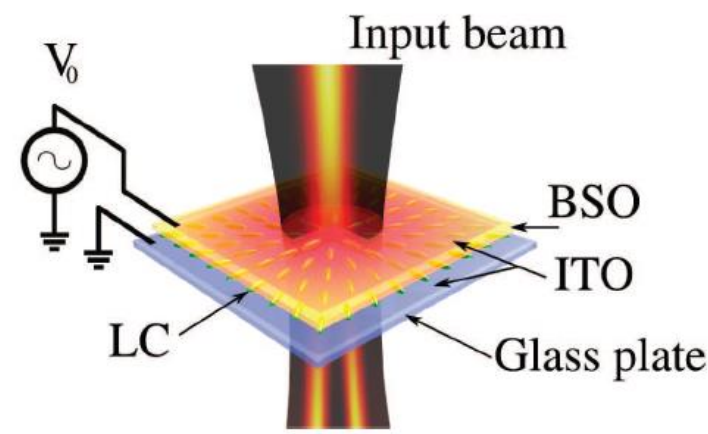

(a)

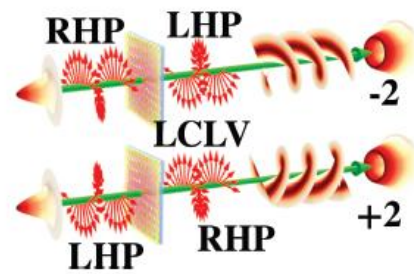

(b)

Fig. 6.5 [53]. Illustration of the self induction mechanism in the LCLV. A Gaussian beam sent on the BSO, will exit with a doughnut profile, an optical vortex with topological charge $m$ such that $|m|=2$. The phase helicity depends on the helicity of the input beam which can be either +1 (left handed polarized beam) or -1 (right handed polarized beam).

It is possible to generate optical vortices using the so-called Panchnaratnam-Berry phase optical elements (PBOEs) on the base of anisotropic media [47]. Such PBOEs are created by the spatial manipulation polarization of an $\mathrm{M}$ wave for the change of a wave front and providing, in such a manner, the coupling of the spin and orbital angular momenta, what is achievable just in an anisotropic medium. To obtain this effect, one uses so-called q-plates, i.e. medium which is both anisotropic and inhomogeneous. Qplate is a device, currently realized using LCs, polymers or sub-wavelength gratings. These are the peculiar wave plates with some phase shift between the ordinary and extraordinary beams unchanged under beam propagation. At the same time, polarization at the output will vary according to the relative orientation of the input polarization and the fast axis [53].

Anisotropy of nematic LCs leads to combining the spin and orbital angular momenta in PBOEs. In the case of circularly polarized singularity-free beam, one obtains at the output of a LCLV without defects the beam with polarization orthogonal to the initial one. For that, the topological charge of the generated vortex is determined by the 
polarization of the incident beam. The defect is generated by a field of low frequency imposed on a photoconductive (BSO) layer. The vortices generated into nematic LCs have found out numerous applications in singular optics, as the key parameter in this case is the topological singularity bearing information on a phase of EM wave [53].

For many years, development of optical instruments and technologies presumed the use of ideal (defect-free) monocrystals. However, just the structural defects, such as points or lines where the orientation or translation order of solids or LCs is violated lie in the base of photonic applications providing the optical flows control [54]. The photon crystals and photon-crystal fibers are among these structures [54].

The presence of the defects in crystals associated with electro-optical of photonic peculiarities of crystals determines applications of them, though reliable means of the control of defects in a matter using low-power optical radiation are absent. So, the defects in liquid crystals manifest themselves as a consequence of the phase transitions, breaking symmetry or mechanical tensions. The defects of LCs are associated with specific spatial structure of the molecular director (the optical axis for the uniaxial LCs) and the corresponding refractive-index patterns.

Non-contact control of the structure of a matter using a light and, in its turn, the control of a light using the ordered materials provide wider feasibilities for the development of means for control and patterning of defects in ordered materials and in the optical phase of laser beams. Laser beams supporting the phase singularities can be used for the control of topological structure of LC materials [54].

For generation of the polarization singularities one can use the quartz crystals, $\mathrm{SiO}_{2}$, which are not undergone to torsion stresses. As a plane wave propagates through the optical axis of such crystal, the polarization singularities of two kinds appear due to residual birefringence resulting from the residual mechanical tension. Such crystal is a biaxial one, and the singularity kernel corresponds to the orientation of the optical axes. If the optical axis is oriented at some angle to the direction of beam propagation, the singularity kernel is characterized by non-zero phase difference. In this case, the form of an optical indicatrix is typical for biaxial crystals. The defects of the crystal structure, such as screw dislocations, being the cause of mechanical tension in a medium, provide appearance of the polarization singularities in such crystals [55].

Propagation of a singularity-supporting beam through a crystal results in changing vortex structure. Even small change of the crystal parameters leads to crucial transformation of a phase singularity. For that, if a beam propagates perpendicularly to the optical axis of the same crystal $\mathrm{SiO}_{2}$, conservation of a vortex is accompanied by the transformation of the OAM. The change of the beam structure is accompanied by spatial depolarization caused by changing the spin angular momentum, the beam ellipticity due to changing the orbital angular momentum as well as generation of optical vortices and polarization singularities. In anisotropic media, changing refraction indices for the ordinary and extraordinary beams leads to the changing spin angular momentum. However, different components of a beam are undergone to different transformations. The spatial depolarization results in decreasing spin angular momentum, whole the polarized components are also transformed. These processes are partially compensated, in contrast to the case when a beam propagates along the optical axis, being accompanied by compensation of any transformation of the spin angular momentum. 
The complex polarization distribution is always associated with the polarization singularities, where one of two orthogonal circularly polarized components vanishes, i.e. the corresponding components are characterized by a vortex structure. So, if a uniaxial crystal rotates in respect to the optical axis, vortices moves and their geometry changes. The intensity minimum is enlarged up to division into two vortices of opposite signs. Both vortices propagate independently, and under annihilation of them at the periphery one observes nucleation of a new vortex of the opposite sign at the center of a beam. This process is repeated at the each $90^{\circ}$ crystal rotation [56].

New step in developing singular optics consisted in establishing of nonlinear singular optics realized in nonlinear media with optical singularities, such as optical solitons, in part optical vortex solitons [57-59]. One of the possibilities of generation of a single or double optically dark polychromatic vortex soliton is the use of a $\mathrm{LiNbO}_{3}$ crystal as a medium with a nonlinear response [57].

New branch of nonlinear singular optics is the dynamical singular optics of generic developing singular optical fields what deals with the processes in nonlinear media. $\mathrm{LiNbO}_{3}$ is the conventional medium for generation of the polarization singularities through the induced chain reactions at elliptically polarized speckle fields due to photorefractive 'optical damage effect' [60]. The polarization singularities are generated by the topological chain reactions at speckle fields controlled by photorefractive nonlinearities induced by the probing laser beam. Irrespectively of the type of an optical singularity ( $C$ points, optical vortices or 'optical diabolos' [60]), singularities are characterized by some instant topological structure of the outgoing wave front governing by the sign laws of singular optics. So, in nonlinear media kind of photorefractive LiNbO3:Fe singularities are developed in tangled way by six topological chain reactions driven by nonlinear processes: C-points and optical diabolos for right (left) polarized components domains with orthogonally left (right) polarized optical vortices underlying them [60].

The optical diabolos which arrange major and minor axes of polarization ellipses surrounding circularly polarized C points in two-cone structure [61-64] are of especial interest. Optical diabolos arrangement and transformations give the most useful information about topology of generic developing light fields. Nucleation (annihilation) of optical diabolos and direct-feedback transformations of hyperbolics and elliptics changes instantaneously shape of the corresponding speckle wave front [60].

Usage and measurement of optical diabolos (Fig. 6.6) occur helpful, because they give comprehensive information about topology of wave front of arbitrary complexity, and its transformations because shape and location of hyperbolics and elliptics on a speckle differ crucially.

For measurement and analysis of topology of a speckle field one implements the dynamic Stokes-polarimetry. Spatial-temporal information on peculiarities of evolution of an optical field can be obtained proceeding from existence of the 'islands of stability' and their parameters in developed speckle fields [60]. 


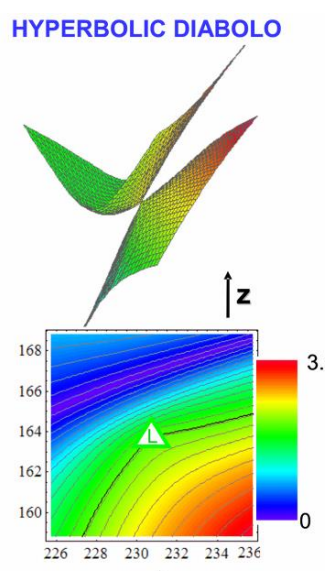

a)

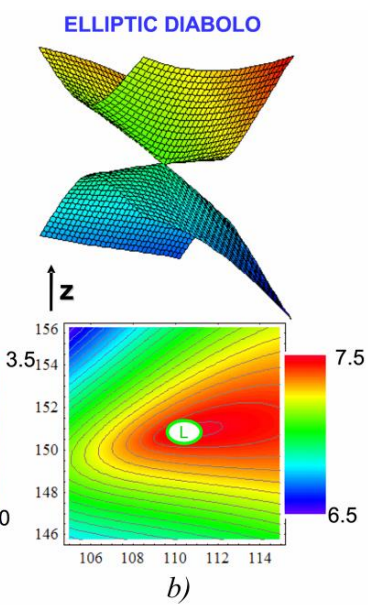

Fig. 6.6 [60]. Hyperbolic (a) and elliptic (b) optical diabolos with two cones of polarization ellipses axes up and below $C$ point. Upper (lower) cone corresponds to distribution of major (minor) axis of polarization ellipses in each point of wave front. The Poincaré index (IP) of hyperbolic (elliptic) diabolo equals $0(+1)$. It is seen a part of thick level height contour of shown hyperbolic which embraces top of the local speckle. Elliptic is always located on the top of a speckle. Triangle (ellipse) marks hyperbolic (elliptic) diabolos. Initial letter $L$ marks lemon morphological form of long axes arrangement in polarization ellipses surrounding $\mathrm{C}$ point.

\subsection{The use of anisotropic media for transformation of singular beam polarization}

Anisotropic media are successfully used for transformation of homogeneously polarized light beams into cylindrical vector beams. So, in part, under weak focusing of a beam in special optical arrangement including a crystal of Iceland spar, the ordinary and extraordinary beams are mixed, that results in forming the beam with the mixed "spiral" polarization. Under stronger focusing, two pronounced focuses are formed. For that, if the initial beam has not a vortex phase, one obtains the polarizations of spiral type; in presence of the vortex phase into initial beam leads to forming pure radial polarization at one of focuses and pure azimuthal polarization at other focus [65].

One of the key problems of modern singular optics consists in control of properties of non-diffracting beams, in part optical vortices inherent in such beams. Anisotropic crystals where the vector nature of the propagating beams must be necessarily taken into account are proper media for this purpose. Vector properties of non-diffracting beams, such as the various Bessel beams propagating in homogeneous, isotropic medium, manifest themselves in inhomogeneous at transversal cross section polarization. Using the singular optics approaches for description of Laguerre-Gaussian and Bessel-Gaussian beams into uniaxial crystals, one can analyze the patterns of conical refraction at these vortex beams and demonstrate of birth and annihilation of optical vortices. Special spectrally integral technique proposed in papers $[66,67]$ ensures the analysis of the Bessel beams propagating through biaxial crystal along the optical axis.

Complex laser modes characterized by the OAM are also of especial interest in crystal optics. There are the optical vortices kind of LG modes having the orbital angular momentum and the beams with various degree of elliptical polarization having the spin angular momentum. Low-order LG beams provide the exchange of the OAM in the lightmatter interaction, in optical tweezers, optical computing, telecommunications, and processing of astronomical images. 
Optical vortices are located at special points where the EM goes to zero and around which the phase distribution forms a complex spiral characterized by the corresponding topological charge. In low-order LG beams, a single optical vortex corresponds to a phase singularity on the beam axis. The use of spiral phase plates (so-called q-plates), plane elements with specified radial orientation of the director at nematic LCs or diffraction elements enable to form the vortices with complex phase distributions.

As it has been mentioned above, the use of light-valve on the base of nematic LC enables to realize self-induction of vortex-beams which possess the ability for selfregulation in respect to the incident light beam. This approach is successfully used to form densely packed lattices of optical vortices with the geometrical structure of a lattice controlled by electrical voltage applied to the light-valve. Correspondingly, the lattice structure and LC defects determine the charge and the OAM of the generated vortex [68].

Thus, anisotropic media forming the vortex beams ensure the possibilities to design optical traps used for manipulation with dielectric microobjects as well as with microobjects with polystyrene, quartz, and silica, to biological systems. Transfer of the OAM from a beam to an object provides arising and development of such manipulations. At the same time, the presence of the spin angular momentum of a circularly polarized light beam propagating through birefingent medium is caused by changing state of polarization. For that, a birefringent crystal, such as calcite, changing the circular polarization of a beam, acquires the torque in the direction opposite to the light polarization (handedness).

The spatial distribution of the state of polarization of a laser beam resulting from propagation through inhomogeneous or anisotropic medium, such as optical fibers or birefringent crystals, being ordered or disordered, determines the feasibilities for using such beams in optical systems. So, laser beams with complex spatial distributions of the states of polarization are the object of active investigations, as unconventional focusing and propagation of them provide new feasibilities for data coding transfer and processing. The radially and azimuthally polarized beams are among such beams. The radially polarized beams are characterized by the axial symmetry, as the electrical vector of a field is radial to the optical axis at the each point of the beam's transversal cross section. In contrast, the azimuthally polarized beams are characterized by the electrical vector of a field perpendicular to the radial distribution at the each point of a cross section. Besides, there is one more kind of beams introduced by Gori [69], viz. the spirally polarized beams combining the properties of the beams of two mentioned kinds.

Radially and azimuthally polarized beams are obtained, in general, at light fields characterized by inhomogeneous polarization distributions formed at propagation of interference combinations of the orthogonally polarized plane beams or linearly polarized Gaussian beams through nematic LCs. The use of the technique based on illumination of homogeneous uniaxial crystal by circularly polarized higher-order Gaussian beam [70,71] or a Gaussian beam shaped by axicons [72] provides its total conversion into radially or azimuthally polarized beams. For that, both radially and azimuthally polarized beams (TE and TM modes) can be generated in polychromatic light using two conical reflectors and polarizing film. For such beams, the azimuth of polarization is undetermined, or singular, on the optical axis of a beam. There are socalled polarization dislocations inherent in radially polarized of sharply focused beams relating to the phase dislocation of a scalar field, i.e. linearly or circularly polarized components of a vector beam [17]. 
A wave front in the vicinity of optical vortex is specified by a helical structure and OAM. The total angular momentum of a light is also characterized by independent contribution associated with the light polarization, namely with a circular polarization causing the spin angular momentum directed along the optical axis.

Quite other situation is realized, when such beams with inhomogeneous distribution of the states of polarization propagate through a birefringent medium. A possible superposition of radially and azimuthally polarized beams form a vortex beam with uniformly distributed circular polarization. Such vortex beam is characterized by the zero angular momentum, as the orbital and spin angular momenta are of the same amplitudes being opposite in sign. Such beam cannot freely propagate through anisotropic medium. As so, a beam decays into simple modes TE and TM. As an exclusion, one can consider the chiral birefringent crystals, where the combination of TE and TM beams forms the spirally polarized beams. In contrast, a vortex beam with the same signs of the orbital and spin angular momenta cannot be described in terms of the radially and azimuthally polarized beams and is stable in different types of anisotropic media [73].

There are two approaches specifying the change of the state of polarization at propagation of laser beams through birefringent medium. The first of them describes the axial propagation of a beam. The second approach relates to oblique propagation of light with respect to the optical axis of the crystal, cf. Sect 6.4.

A homogeneously polarized beam propagating along the optical axis of a uniaxial crystal can be represented as a superposition of the radially and azimuthally polarized beams where, to say, the x-components of all beams form a field with the azimuthally polarized TE mode (ordinary beam), while the y-components form the radially polarized TM mode (extraordinary beam). If the linearly polarized components are formed at the crystal output, they have the angular dislocations along $x$ - or $y$-axes. If the circularly polarized components of TE and TM modes are formed at the crystal output, they have the single-charged optical vortices with opposite signs of the topological charge.

Propagation of a circularly polarized wave through a crystal in special optical setup leads to formation of a field with $\mathrm{C}$ point whose topological index equals unity, located at the axis, being surrounded by numerous $C$ lines with altering clockwise and counterclockwise circular polarizations. The distribution of polarization singularities can change due to changing positions of elements of the experimental arrangement, for that it is possible to form the doubly charged vortices into orthogonally polarized components. The topological charge of vortex is changed in the opposite one with changing the handedness of the circular polarization in the initial light beam [74]. Birefringent anisotropic media can be also used for formation of complex polarization distributions including polarization singularities with optical vortices in any polarization component. The example of realization of this approach is the use of micron-scale LC droplets without any additional optical elements [74].

Transparent uniaxial crystals generating the doubly-charged optical vortices can conserve the angular momentum of a light, when a circularly polarized beam propagates along the optical axis of a crystal. It is known, that the total angular momentum is conserved for arbitrary direction of propagation of a beam in a free space or in optically homogeneous and isotropic medium. As a rule, a birefringent medium transforms both polarization and form of a beam that results in changing the orbital and 
spin angular momenta of a beam, though the projection of the angular momentum on the beam axis is conserved [75].

Indeed, when a beam propagates through a birefringent medium, the orbital and spin angular momenta are transformed, and the mechanism of the spin-to-orbital conversion is provided due to spin-orbit coupling. This effect has been investigated theoretically and experimentally [74] using uniaxial calcite crystals cut perpendicularly to the optical axis for the monochromatic and polychromatic vortex-free Gaussian beams at the crystal input.

Under propagation of a vortex-free Gaussian beam through a crystal, the depolarizing effects decrease the spin angular momentum flux as a whole. At the same time, conservation of the total angular momentum is accompanied by the polarization states that is connected with generation of doubly-charged optical vortices at the circularly polarized component of a field with the opposite direction of rotation of the electrical vector (hanedness) in respect to the state of circular polarization of the incident beam.

There are numerous paraxial beams whose structure is transformed under propagation, including Hermite-Laguerre-Gaussian and Bessel-Gaussian beams with a complex argument [76]. Under propagation through birefringent crystal, the ring structure is generated consisting of right circularly polarized and left circularly polarized components forming one circle. The resulting intensity distribution is determined by the phase difference of the ordinary and extraordinary beams.

For some thickness of a crystal, the typical conoscopic pattern starts to take shape. For that, the total intensity of the right circular polarization decreases and the total intensity of the left circular polarization increases. Further, formation of the ring structure results from increasing the right-hand circular components, while the left-handed circular component results from decreasing of total intensity. In the course of time, such distribution is set at a whole crystal thickness. The maximal magnitude of intensity takes place, if the phase difference between the ordinary and extraordinary beams within small region corresponds to some intensity ring and the curvature radii of the wave fronts [76]. Note, the spin-to-orbital conversion is not obligatory for standard HG and LG beams with a real argument.

The influence of birefringent crystals on a light flux can be illustrated using the Bessel optical beams, which is represented as a set of conical beams of plane waves.

Propagation of such beam through a chiral uniaxial birefringent crystal is accompanied with the transformation of homogeneously polarized beam into spirally polarized one. As a consequence, the incident beam undergoes considerable inhomegeneous polarization transformations even at short-distance propagation in a crystal. The beam is gradually transformed into regularly spirally polarized, with certain chirality. A propagating beam can also result in formation of spirally polarized beams with the opposite chirality [77, 78].

One meets interesting example of a beam transformation in the system (succession) of two birefringent crystals with chirality of the opposite signs. The crystals are oriented in such a manner that the distribution referred to as the Airy spirals is formed [74]. At propagating the vortex-beams, including the vortex-free Gaussian beam, trough two birefringent chiral crystals with the opposite chirality, one can realize a complex intensity distribution both in monochromatic and polychromatic light [74]. 
In Fig.6.7 [74], the maps of the polarization states are presented for the system of two $\mathrm{SiO}_{2}$ crystals with the opposite signs of chirality that is provided by linearly polarized beam with the electrical vector oriented at the angle $45^{\circ}$ to the axes of the reference frame. The distinguishing sign of these polarization distributions is the vector topological quadrupole consisting of four polarization singularities (two stars and two lemons) located at bends of $L$ lines (the lines with linear polarization). These lines form the system of concentric rings and a double spiral. Further propagation of such spiral-like structure of polychromatic light beam through linear polarizer results in the intensity distribution in the form of a typical Airy spiral pattern (Fig. 6.7(e)).

(a)

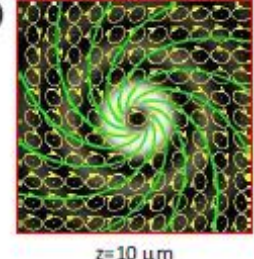
$z=10 \mu \mathrm{m}$

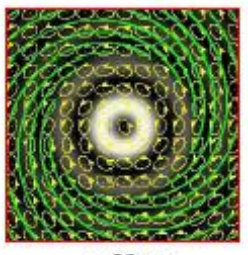

$z=38 \mu \mathrm{m}$

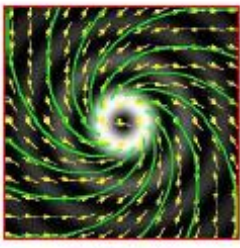

$z=20 \mu \mathrm{m}$

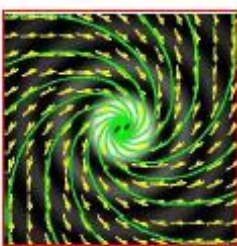

$z=65 \mu \mathrm{m}$

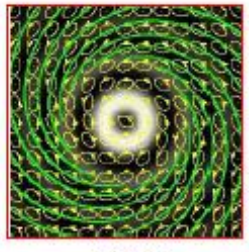

$z=36 \mu \mathrm{m}$

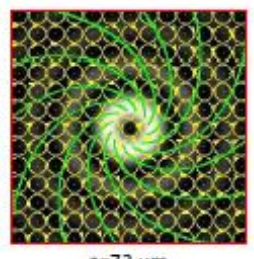

$z=73 \mu \mathrm{m}$
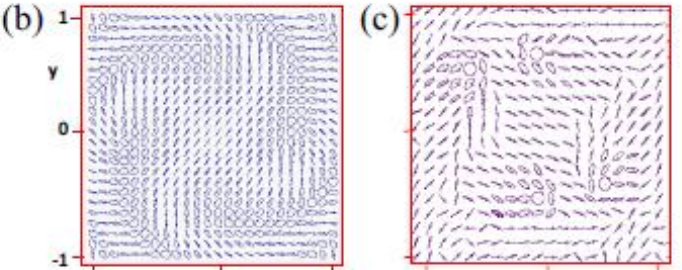

(d)

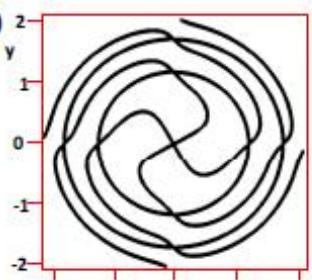

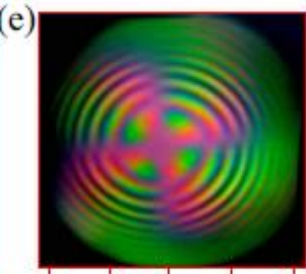

Fig. 6.7 [74]. (a) Maps of the polarization states on the background of the intensity distribution of a Bessel beam; the curves show the directions of the major axes of polarization ellipses. (b) Theoretical and (c) experimental maps of the polarization states, and (d) the theoretical edge dislocation lines for the monochromatic light. (e) Experimental intensity distribution of the polychromatic light from a halogen lamp for the Gaussian beam after the system of two $\mathrm{SiO}_{2}$ birefringent chiral crystals with opposite signs of chirality [78].

The represented results describe propagation of singular beams through uniaxial crystals, for example along the optical axis of c-cut crystal.

Thus, investigations of propagation of light through anisotropic media have leads to tha development of a powerful instrument for spatial engineering of polarization and phase providing all-optical approaches for forming singularities at scalar and vector optical beams. Taking into account the exceptional role of birefringence in formation of peculiar distributions of optical fields, the properties of crystals, such as optical activity and dichroism, has led to reaching the feasibilities for optical manipulation of a wide class of the objects of different origin and properties. In this context, the possibility to form polychromatic ('white-light') vortices is of special importance. Generalization of the discussed approaches to generation of phase and polarization singularities presumes further consideration of propagation of the beams with complex polarization distributions through anisotropic crystals, what is accompanied by changing states of polarization and of the orbital and spin angular momenta of such beams. Non-trivial effects are also observed when cylindrical symmetry of a beam propagating at some angle in respect to the optical axis of a crystal is break. Generally, one can state a trend to considerable increasing the approaches providing capturing and transporting microparticles as well as controlling of their mutual orientation in solving the problems of nanophotonics, nanophysics, precise chemistry and pharmacology, viz. in all practical applications where one deals with the control of the objects of micro- and nano-scales. 


\section{References}

1. Berry M.V., Dennis M.R., "The optical singularities of birefringent dichroic chiral crystals," Proc. R. Soc. Lond. A 459, 1261-1292 (2003).

2. Berry M.V., Bhandari R., Klein S., "Black plastic sandwiches demonstrating biaxial optical anisotropy," Eur. J. Phys. 20, 1-14 (1999).

3. Boulanger P., Hayes M., "Electromagnetic plane waves in anisotropic media: an approach using bivectors," Phil. Trans. R. Soc. Lond. A 330, 335-393 (1990).

4. Born M., Optik: ein Lehrbuch der Elektromagnetischen Lichttheorie, Springer-Verlag, Berlin (1933).

5. Born M., Wolf E., Principles of Optics, ( $7^{\text {th }}$ ed, expanded), Cambridge U. Press, Cambridge (1999).

6. Landau L.D., Lifshitz E.M., Pitaevskii L.P., Electrodynamics of Continuous Media, Oxford: Pergamon (1984).

7. Nye J.F., Physical Properties of Crystals, (2nd ed). Oxford: Clarendon (1995).

8. Ramachandran G.N., Ramaseshan S., Crystal Optics, XXV/I, in Handbuch der

Physik, ed. H. Flügge, Springer-Verlag, Berlin (1961).

9. Nye J.F., "Lines of circular polarization in electromagnetic wave fields," Proc. R. Soc. Lond. A 389, 279-290 (1983).

10. Freund I., Soskin M., Mokhun A.I., "Elliptic critical points in paraxial optical fields," Opt. Commun. 208, 223-253 (2002).

11. Dennis M. R., "Polarization singularities in paraxial vector fields: morphology and statistics," Opt. Commun. 213, 201-221 (2002).

12. Freund I., "Second harmonic generation of optical ellipse fields," Opt. Commun. 213, 129-149 (2002)

13. Nye J.F., Berry M.V., "Dislocations in wave trains," Proc. R. Soc. Lond. A 336, 165190 (1974).

14. Optical Vortices, eds: M. Vasnetsov, K. Staliunas, New York: Nova Science (1999).

15. Berry M.V., "Much ado about nothing: optical dislocation lines (phase singularities, zeros, vortices ,...)," Proc. SPIE 3487, 1-5 (1998).

16. Berry M.,V., Upstill C., Catastrophe Optics: Morphologies of Caustics and Their Diffraction Patterns, In: Progress in Optics, ed. E. Wolf, 18, 257-346 (1980).

17. Nye J.F., Natural Focusing and Fine Structure of Light: Caustics and Wave

Dislocations, Institute of Physics Publishing, Bristol and Philadelphia (1999).

18. Fadeyeva T.A., Rubass A.F., Sokolenko B.V., Volyar A.V., "The precession of vortex-beams in a rotating uniaxial crystal," Journ. Opt. A: Pure Appl. Opt. 11, 53-55 (2009).

19. Stamnest J.J., Sherman G.C., "Radiation of electromagnetic fields in uniaxially anisotropic media," J. Opt. Soc. Am. 66, 780-788 (1976).

20. Carrier G.F., "On the nonlinear vibration problem of the elastic string," Quart. Appl. Math. 3, 157-165 (1945).

21. Bunkin F.V., "On radiation in anisotropic media," JETP 5, 277-295 (1957).

22. Lighthill M. J., "Studies on magneto-hydrodynamic waves and other anisotropic wave motions," Phil. Trans. R. Soc. Lond. A 252, 397-430 (1960).

23. Fleck J.A., Feit M.D., "Beam propagation in uniaxial anisotropic media," J. Opt. Soc. Am. 73, 921-926 (1983).

24. Ciattoni A., Palma C., "Optical propagation in uniaxial crystals orthogonal to the optical axis: paraxial theory and beyond," J. Opt. Soc. Am. A 20, 2163-2171 (2003).

25. Volyar A.V, Fadeyeva T.A., "Generation of singular beams in uniaxial crystals," Opt. Spectrosc. 94, 333-345 (2003).

26. Volyar A.V, Yegorov Yu.A., Rubass A.F ., Fadeyeva T.A., "The fine structure of optical vortices in a crystal: a monochromatic beam," J. Techn. Phys. 74, 90-93 (2004). 
27. Berry M.V., Hannay J. H., "Umbilic points on Gaussian random surfaces," J. Phys. A: Math. Gen. 10, 1809-1821 (1977).

28. Ciattoni A., Cincotti G., Palma C., "Circular polarized beams and vortex generation in uniaxial media," J. Opt. Soc. Am. A 20, 163-171 (2003).

29. Volyar A., Fadeyeva T., "Optical vortices in crystals: formation, annihilation and decay of polarization ombilics," Techn. Phys. Lett. 29, 111-114 (2003).

30. Volyar A. and Fadeyeva T., "Laguerre-Gaussian beams with complex and real arguments in uniaxial crystals," Opt. Spectrosc. 101, 297-304 (2006).

31. Vasilev V.I., Soskin M. S., "Topological scenarios for the production and annihilation of polarization singularities in nonstationary optical fields," JETP Lett. 87, 90-93 (2008).

32. Berry M., Dennis M., "The singularities of crystal optics," ICO Topical Meeting on Polarization Optics, University of Joensuu, 18-19 (2003).

33. Flossmann F., Schwarz U. T., Dennis M.R., "Polarization singularities from unfolding an optical vortex through a birefringent crystal," Phys. Rev. Letts. 95, 253901 - 1-4 (2005).

34. Dennis M.R., "Polarization singularities in paraxial vector fields: Morphology and statistics," Opt. Commun. 213, 201-221 (2002).

35. Soskin M., Denisenko V., Egorov R., "Topological networks of paraxial ellipse speckle fields," J. Opt. A 6, S281-S287 (2004).

36. Nesci A., Dandliker R., Salt M., Herzig H.P., "Measuring amplitude and phase distribution of fields generated by gratings with sub-wavelength resolution," Opt.

Commun. 205, 229 - 238 (2002).

37. Fadeyeva T.A., Volyar A.V., "Nondiffracting vortex-beams in a birefringent chiral crystal," https://arxiv.org/ftp/arxiv/papers/0910/0910.5909.pdf.

38. Fadeyeva T., Rubass A., Egorov Yu., Volyar A., Shvartzlander G., "Quadrefringence of optical vortices in a uniaxial crystal,", J. Opt. Soc. Am. A 25, 1634-1641 (2008).

39. Fadeyeva T., Rubass A., Egorov Yu., Volyar A., Swartzlander Gr.,

"Quadrefringence of optical vortices in a uniaxial crystal, ," J. Opt. Soc. Am. A 25, 16341641 (2008).

40. Volyar A., Shvedov V., Fadeyeva T., Desyatnikov A. S., Neshev Dr.N., Krolikowski W., Kivshar Yu.S., "Generation of single-charge optical vortices with an uniaxial crystal," Opt. Expr. 14, 3724-3729 (2006).

41. Fadeyeva T., Rubass A., Volyar A., "The transverse shift of a high order paraxial vortex-beam induced by a homogeneous anisotropic medium," Phys. Rev. A 79, 053815 - 1-12 (2009).

42. Brasselet E., Izdebskaya Ya., Shvedov V., Desyatnikov A., Krolikowsky W., Kivshar Yu., "Dynamics of optical spin-orbit coupling in uniaxial crystals," Opt. Lett. 34, 10211023 (2009).

43. Fadeyeva T.A., Rubass A.F., Volyar A.V., "The matrix model of vortex-beam quadrefringence in a uniaxial crystal," Ukr. J. Phys. Opt. 10, 109-123 (2009).

44. Berry M., Klein S. "Colored diffraction catastrophes," Proc. Natl. Acad. Sci. USA. Physics 93, 2614-2619 (1996).

45. Volyar A., Yegorov Yu.A., Rubass A.F, Fadeyeva T.A., "Fine structure of "white" optical vortices in a crystal," JTP Letts. 30, 82-89 (2004).

46. Volyar A.V., Fadeyeva T.A., "Generation of singular beams in uniaxial crystals," Opt. Spectrosc. 94, 2, 260-270 (2003)

47. Egorov Yu.A., Fadeyeva T.A., Volyar A.V., "The fine structure of singular beams in crystals: colours and polarization," J. Opt. A: Pure Appl. Opt. 6, S217-S228 (2004). 48. Berry M.V., Jeffrey M.R., Mansuripur M., "Orbital and spin angular momentum in conical diffraction," J. Opt. A: Pure Appl. Opt. 7, 685-690 (2005). 
49. Angelsky O.V., Besaha R.N., Mokhun I.I., "Appearance of wavefront dislocations under interference among beams with simple wavefronts," Proc.SPIE, 3317, 97-100 (1997).

50. MacDonald A.T., O'Brien A.E., Herne C.M., "Manipulation of calcite crystals in a beam with a polarization singularity," Frontiers in Optics/Laser Science, JTu4A.50 (2015).

51. Izdebskaya Y., Brasselet E., Shvedov V., Desyatnikov A, Krolikowski W., Kivshar Yu., "Dynamics of linear polarization conversion in uniaxial crystals," Opt. Expr. 17, 18196-18208 (2009).

52. Brasselet E., Murazawa N., Misawa H., and Juodkazis S., "Optical vortices from liquid crystal droplets," Phys. Rev. Letts., 103, 103903 - 1-4 (2009).

53. Barboza R., "Spatial solitons and optical vortices in nematic liquid crystals," PhD Thesis (2014).

54. Smalyukh I., Ackerman P., Trivedi R. P., Lee T., "Control of matter defects using optical phase singularities," SPIE Newsroom, DOI: 10.1117/2.1201008.003079 (2010). 55. Vasylkiv Yu., Savaryn V., Smaga I., Skab I., Vlokh R., "Polarisation singularities of optical beam propagating in imperfect quartz crystals," Ukr. J. Phys .Opt. 13, 204-206 (2012).

56. Sokolenko B.V., Rubass A.F., Volyar A.V., "Generation of optical vortices in rotating uniaxial crystal for light propagation along the perpendicular to its optical axis,"

Semicond. Phys, Quantum Electr. \& Optoelectron 16, 344-348 (2013).

57. Neshev D., Dreischuh A., Shvedov V., Desyatnikov A. S., Krolikowski W., Kivshar Yu. S., "Observation of polychromatic vortex solitons," Opt. Lett. 33, 1851-1853 (2008). 58. M S Soskin M.S., Vasnetsov M.V., "Nonlinear singular optics," Pure Appl. Opt. 7, 301-312 (1998).

59. Jeng C.C., Ming-Feng Shih M.-F., Motzek K., Kivshar Yu., "Partially incoherent optical vortices in self-focusing nonlinear media," Phys. Rev. Lett. 92, $043904-1-4$ (2004).

60. Vasil'ev V.I., Soskin M.S., "Tangled nonlinear driven chain reactions of all optical singularities," https://arxiv.org/ftp/arxiv/papers/1201/1201.1537.pdf (2012).

61. Egorov R.I., Soskin M.S., Freund I., "Experimental optical diabolos," Opt. Lett. 31, 2048-2050 (2006).

62. Freund I., Soskin M.S., Egorov R.I., "Diabolo creation and annihilation," Opt. Lett.

31, 2381-2383 (2006).

63. Soskin M.S., Egorov R.I., Freund I., "Experimental umbilic diabolos in random optical fields," Opt. Lett. 32, 891-893 (2007).

64. Freund I., "Optical diabolos: Configurations, nucleations, transformations and reactions," Opt. Commun. 272, 293-309 (2007).

65. Khonina S.N., Karpeev S.V., Alpherov S.V., "Theoretical and experimental investigation of polarization transformations in uniaxial crystals for obtaining high-order cylindrical vector beams," Computer Optics 38, 171-180 (2014).

66. Barboza R., Assanto G., Bortolozzo U., Clerc M.G., Residori S., Vidal-Henriquez E., "Programable lattices of optical vortices in nematic liquid crystal," Proc SPIE 9565, 95650L - 1-7 (2015).

67. Belyi V., King T., Kazak N., Khilo N., Ryzhevich A., Katranji E., "Methods of formation and nonlinear conversion of Bessel optical vortices," Proc. SPIE 4403, 229240 (2001).

68. Kazak N., Khilo N., Ryzhevich A., "Generation of Bessel beams under the conditions of internal conical refraction," Quant. Electron. 29, 1020-1024 (1999).

69. Gori F., "Polarization basis for vortex beams," J. Opt. Soc. Am. A 18, 1612-1617 (2001). 
70. Fadeyeva T., Kotlyarov K., Volyar A., "Extreme spin-orbit coupling in HermiteGaussian beams in a uniaxial crystal," arXiv:0902.3716 (2009).

71. Fadeyeva T., Volyar A., "Extreme spin-orbit coupling in crystal-travelling paraxial beams," J. Opt. Soc. Am. A 27, 381-389 (2010).

72. Loussert C.,

Brasselet E., "Efficient scalar and vectorial singular beam shaping using homogeneous anisotropic media," Opt. Lett. 35, 7-9 (2010).

73. C. Alexeyev, A. Volyar, M. Yavorsky, Fiber Optical Vortices, In: Lasers, Optics and Electro-Optics Research Trends, ed. L. I. Chen, Nova Science Pub., 131-223 (2007). 74. Fadeyeva T.A., Shvedov V.G., Izdebskaya Ya.V., Volyar A.V., Brasselet E., Neshev D.N., Desyatnikov A.S., Krolikowski W., Kivshar Yu.S., "Spatially engineered polarization states and optical vortices in uniaxial crystals," Opt. Expr. 18, 10848-10863 (2010).

75. Ciattoni A., Cincotti G., Palma C., "Angular momentum dynamics of a paraxial beam in a uniaxial crystal," Phys. Rev. E 67, 036618 - 10-109 (2003).

76. Volyar A., Fadeyeva T., "Laguerre-Gaussian beams with complex and real arguments in uniaxial crystals," Opt. Spectrosc. 101, 297-304 (2006).

77. Fadeyeva T., Volyar A., "Nondiffracting vortex-beams in a birefringent chiral crystal," J. Opt. Soc. Am. A 27, 13-20 (2010).

78. Rubass A., Fadeyeva T., Egorov Yu., Shvedov V., Volyar A., Desyatnikov A.S., Kivshar Yu.S., "Spiral-like singular beams in gyrotropic crystals," Proc. SPIE 6254, $62540 \mathrm{H}-1-8$ (2006). 